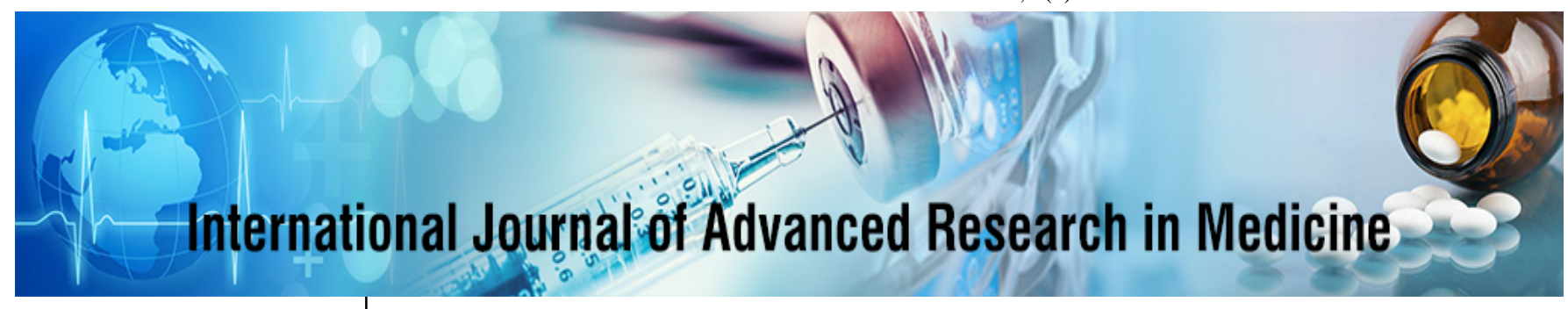

E-ISSN: 2706-9575

P-ISSN: 2706-9567

IJARM 2020; 2(2): 193-197

Received: 29-08-2020

Accepted: 10-09-2020

Nemaa Mazyed Abdulazeez MBChB DA (Anesthetist), Ministry of Health, Medical City Baghdad Teaching Hospital, Baghdad, Iraq

Aysar Abd Oun Ali Al-Zubaidi MBChB DA CABA and IC (Anesthetist), Head of Department of Anesthesia, Ministry of Health, Baghdad Medical Office - Al-Karkh, Al Karkh General Hospital, Baghdad, Iraq

Abeer Kamal Husein Alkashab MBChB DA (Anesthetist), Ministry of Health, Baghdad Medical Office - Al-Karkh, Al Karama Educational Hospital, Baghdad, Iraq
Corresponding Author: Nemaa Mazyed Abdulazeez MBChB DA (Anesthetist), Ministry of Health, Medical City Baghdad Teaching Hospital, Baghdad, Iraq

\section{Giving anesthesia for open heart surgery}

\author{
Nemaa Mazyed Abdulazeez, Aysar Abd Oun Ali Al-Zubaidi and Abeer \\ Kamal Husein Alkashab
}

DOI: https://doi.org/10.22271/27069567.2020.v2.i2c.69

\begin{abstract}
Cardiopulmonary bypass (CPB) required for cardiac surgery presents unique challenges to the cardiac anesthesiologist who is responsible not only for maintaining hemodynamics but at the same time anesthesia and analgesia for these patients. Unique pathophysiologic changes during CPB result in pharmacokinetic alterations that impact the serum and tissue concentrations of IV and volatile anesthetics. CPB has evolved into routine, safe and reliable system that requires intensive specialized training to operate. It is the function of the percussionist to maintain and operate this equipment during $\mathrm{CPB}$; however anesthesiologist and surgeon caring for the patient undergoing CPB bear the responsibility of understanding this equipment and its function in order to manage the patient undergoing surgical procedure safely. Anesthetic management of patient coming for cardiac surgery and different component of cardiopulmonary bypass will be discuss in this review article.
\end{abstract}

Keywords: Anesthesia; cardiac surgery; cardiopulmonary bypass; extracorporeal circulation

Citation: Samad K, Hashmi M. Anesthetic Management for Open Heart Surgery and Extracorporeal Circulation. Anaesth Pain \& Intensive Care. 2016;20 Suppl 1:S140-S145

\section{Introduction}

Anesthetic management of patient coming for cardiac surgery is challenging and highly demanding. Improvements in hemodynamic monitoring, myocardial protection and surgical and anesthesia techniques have led to improved outcome of these high risk patients. Commonly performed procedures on cardiopulmonary bypass (CPB) include coronary artery bypass grafting (CABG), valve repair or replacement, palliative or corrective procedures for congenital heart disease, major vessels repair or replacement and heart transplantation.

\section{Assessment of cardiac risk}

Various scoring systems have evolved over the years to quantify the risk of cardiac surgery. However, these scores can only determine the likelihood of mortality in a large number of patients and are not sensitive enough to predict the outcome of individual patients. European System for Cardiac Operative Risk Evaluation (EuroSCORE) has been validated across different centers in Europe and across the world ${ }^{[1]}$. The Cardiac Anesthesia Risk Evaluation Score (CARE Score) simplifies the approach to assess risk of cardiac surgical procedures in a manner similar to the original ASA physical status classification and demonstrates similar or superior predictive characteristics compared with the more complex indices. ${ }^{2}$

\section{Determinants of perioperative myocardial injury in cardiac surgery 1. Disruption of coronary blood flow}

Duration of aortic cross clamping (AXC) and cardiopulmonary bypass (CPB) time has consistently shown to be the main determinants of postoperative outcome in virtually all studies.

\section{Reperfusion of ischemic myocardium}

Reperfusion after interruption of blood flow is essential for tissue survival, but cell damage can occur as a result of reperfusion itself. ${ }^{5}$ Myocardial ischemia of less than 20 minutes, followed by reperfusion, is characterized functionally by transient contractile dysfunction without evidence of structural injury. This is called MYOCARDIAL STUNNING which eventually recovers completely, especially with the help of inotropes. 
But reperfusion of cardiac tissue subjected to an extended period of ischemia, results in irreversible myocardial injury or cellular necrosis. This is known as 'Myocardial Reperfusion Injury' and is defined as the death of myocytes alive at the time of reperfusion, as a direct result of one or more events initiated by reperfusion. The extent of tissue necrosis that develops during reperfusion is directly related to the duration of the ischemic event

\section{Myocardial preconditioning}

This is a phenomenon in which exposure to a brief stimulus can reduce the subsequent injury from a sustained period of lethal ischemia followed by reperfusion ${ }^{[3]}$. Preconditioning can be induced by repetitive episodes of brief ischemia, volatile anesthetic agents and morphine and antagonized by hyperglycemia.

\section{Anesthetic management}

The goals of anesthesia for cardiac surgery include avoidance of perioperative cardiac ischemia, tight hemodynamic control, early extubation and avoidance of non-cardiac complications. Obsessive attention to detail enhances patient safety and outcome.

\section{Preoperative evaluation}

Patients scheduled for cardiac surgery are usually fully investigated for their cardiac status. However, the presence of non-cardiac medical illnesses and routine general anesthetic assessment should not be overlooked including routine laboratory investigation and arrangements of blood products. Acute unstable angina, acute MI ( $<7$ days), CCF, cardiogenic shock, emergency surgery, re-do surgery and combined procedures present the highest risk. Reversible defects on the thallium scan represent viable myocardium supplied by a diseased coronary artery which will benefit from revascularization. On Echocardiography an EF > 40\% represents low risk, 30\%-40\%; intermediate risk, < 30\% high risk. Presence of acute VSD, ventricular aneurysm and MR due to papillary muscle rupture indicate severe disease and increased risk. Left main and proximal LAD lesions in addition to distal lesion on three vessels on coronary angiogram present the highest risk.

Premedication may help prevent preoperative anginal episodes (secondary to hypertension and tachycardia) and include anti-anginal medications, especially beta blockers if the patient is already taking them. Over sedation and hypoxia should be avoided. Most cardiac medications are continued up until the morning of surgery including $\beta$ blockers which reduces myocardial oxygen demand and also the risk of arrhythmias. However, ACE-inhibitors should be discontinued as continued use may increase the risk of hypotension during induction.

\section{Monitoring}

It is mandatory to have an arterial line prior to induction of anesthesia in high risk cases such as left main stem coronary artery stenosis. Central venous access is required for measuring CVP and vasoactive drug administration. Except high risk cases it is done after induction. Despite the substantial amount of physiologic information obtained from pulmonary artery catheterization, mortality is not influenced by its routine use during cardiac surgery and may be increased due to the potential arrhythmogenic effects of the catheter. Use of PAC should be reserved for high risk cases (EF <40\%, CCF or RWMA), valve surgery, complex procedure and for combined procedures by experienced anesthesiologists in centres familiar with its use. Transoesophageal echocardiogram (TOE) provides continuous monitoring of regional and global ventricular function, myocardial ischemia, volume status and post valve repair or replacement status. ${ }^{5}$ Patients undergoing cardiac surgery are at a higher risk for intraoperative awareness or recall and Bispectral Index (BIS) is one of the several technologies used to monitor the depth of anesthesia. A significant reduction in awareness during cardiac surgery may occur when the BIS is monitored. ${ }^{6}$ Stroke occurs in 1$3 \%$ of patients following $\mathrm{CPB}$ and neuropsychological complications may be found in up to $70 \%$ patients. Cerebral Function Analysing Monitor (CFAM), Transcranial Doppler (TCD) and Near Infra-red spectroscopy (NIRS) do not reliably detect cerebral insults during $\mathrm{CPB}$ but might be helpful in high risk patients.

\section{Pre-bypass management}

There is no ideal induction agent and there is no place for a 'mono-agent' technique for induction of general anesthesia. Various combinations of opioids and benzodiazepines and other intravenous induction agents can be used. 'It is not what is used, but how it is used to ensure hemodynamic stability that is important'. There is increasing evidence for preconditioning effects of volatile agents and their liberal use is encouraged.

Nitrous oxide should be avoided due to its negative inotropic effect and possible expanding effects on cerebral or coronary gaseous emboli. Antifibrinolytic agents are used to reduce perioperative blood loss and transfusion requirements. Aminocaproic acid has variable efficacy and concerns of renal vasoconstriction and thrombosis lack clinical significance. Recent concerns regarding renal failure has led to reduced use of Aprotinin. There is strong evidence in literature for the safety and efficacy of Tranexamic acid. Depth of anesthesia should be adequate before median sternotomy and supplemented with opioids. The anesthesiologist deflates the lungs before the sternal saw divides the sternum, to reduce the risk of opening the pleura. Adequate systemic anticoagulation is an absolute requirement for CPB. A heparin dose of $300-400$ units $/ \mathrm{kg}$ is given and adequacy of anticoagulation confirmed by an ACT $>400$ sec for institution of CPB. Resistance is usually associated with prolonged preoperative administration and is treated with higher doses of heparin or with FFPs. Mild hypotension due to vasodilatation is common and heparin induced thrombocytopenia may occur. Excessive hypertension (MAP $>80 \mathrm{mmHg}$ ) should be avoided during cannulation of ascending aorta to reduce the risk of localized arterial dissection. Right atrial (RA) cannulation may be accompanied by hemodynamic instability secondary to atrial dysrhythmias, impeded venous return and/or hemorrhage.

\section{Bypass management}

As the venous or the RA cannula is unclamped, RA blood drains into the oxygenator by gravity or siphonage and oxygenated blood is pumped into the aorta. On achieving full flow, mechanical ventilation and all maintenance fluids are discontinued. Acute hemodilution produces an abrupt fall in blood viscosity and systemic vascular resistance (SVR). Consequently, transient hypotension (MAP: 30- 40 
$\mathrm{mmHg}$ ) at the onset of $\mathrm{CPB}$ is common and usually responds to vasoconstrictors. At the onset of cardiopulmonary bypass, circulating drugs are subject to hemodilution, altered protein binding and sequestration, and there is a risk of inadequate anesthesia or 'awareness'. Anesthesia can be maintained by either a propofol infusion (2-4 mg/ kg/hr) or inhalation anesthesia through the CPB by the perfusionist. During the CPB, the heart beats empty, but the coronary arteries are still perfused from the aorta. Clamping the aorta between the site of insertion of the aortic cannula and origin of the coronary arteries produces a bloodless field, a flaccid heart, and avoids air embolism. Myocardial preservation includes the use of cardioplegia given via the aortic root down the coronaries and systemic and topical hypothermia. Cardioplegia stops the electromechanical activity producing diastolic arrest in the ischemic heart, preventing energy consumption and metabolite accumulation. The composition varies but most use $\mathrm{K}+$ as the arresting agent with $\mathrm{Ca} 2+$, glucose, buffers and Mg2+. As the myocardium continues to receive a small amount of non-coronary (bronchial and pericardial) collateral blood flow, cardioplegic solution is gradually washed out over 20-30 min after which further cardioplegia is required.

\section{Post-bypass management}

Rewarming should be gradual to avoid excessive thermal gradients. Nasopharyngeal temperature monitoring may underestimate cerebral venous temperature by up to $3.4^{\circ} \mathrm{C}$ when rewarming is very rapid and as little as $0.5^{\circ} \mathrm{C}$ hyperthermia may exacerbate ischemic CNS injury. Inadequate rewarming on the other hand, combined with further heat loss during chest closure, results in shivering, vasoconstriction, increased oxygen consumption and coagulopathy. Prolonged ventricular fibrillation (VF) and LV distension may occur during rewarming and coronary reperfusion following removal of aortic clamp. The increase in myocardial oxygen consumption may cause subendocardial ischemia. Defibrillation is done with 10-20J monophasic shock delivered by internal 'paddles'. Inotropes and vasoactive drugs use should be planned depending on the presence of predictors for difficulty in weaning from CPB. In the absence of systemic factors like electrolyte imbalance (hyperkalaemia) and acid base disturbance, failure to wean from $\mathrm{CPB}$ is most likely from intraoperative myocardial injury or cardiac dysfunction. A checklist should be available before coming off $\mathrm{CPB}$ to make sure all the parameters are checked including normal sinus rhythm, temperature, serum potassium level, hemoglobin levels etc. Intra-abdominal Balloon Pump (IABP) counter-pulsation is used when inotrope therapy alone is insufficient to maintain hemodynamic stability. IABP counter-pulsations augment diastolic coronary perfusion and forward flow. Epicardial leads are placed in atria and ventricle to maintain heart rate at $80-90$ beats per minute to avoid ventricular distention in high-risk cases. However, use of epicardial leads is associated with a small risk of cardiac damage during insertion, and hemorrhage following removal. Protamine is empirically administered as a slow infusion (1mg for each 100 units of heparin given) after a review article test dose. As the surgeon secures hemostasis the anesthesiologist makes sure the ACT returns to base line, and appropriate blood products are infused depending on the laboratory results of hematocrit, platelet count and coagulation profile. Chest tubes are placed and attached to an under-water seal.

\section{Off-pump surgery}

To avoid the adverse effects of the heart-lung machine, coronary surgery can be performed on a beating heart using special devices to steady the part of the heart the surgeon is working on. There is no cannulation, extracorporeal circulation or cross- clamping of the aorta. However, the procedure is more demanding for the anesthesiologist who has to maintain hemodynamic stability as the heart is mobilized, retracted and stabilized.

\section{Fast-track patients}

Early extubation (within 6 hours) in low risk patients who are not bleeding and don't require inotropic support is safe. Early extubation reduces the length of hospital stay and cuts the cost of uncomplicated cardiac surgery without compromising standards of care. On-table extubation is possible but has not been demonstrated to be of clinical benefit.

\section{The cardiopulmonary bypass circuit}

The heart-lung machine provides mechanical circulatory support and replaces the function of heart and lungs. The machine component consists of cannulae (venous and arterial), tubing (polyvinyl or silicone), reservoir, oxygenator (bubble or membrane), cardiotomy (filtered reservoir), heat exchanger(s), arterial line filter, pump(s), flow meter, inline blood gas and electrolyte analyzer, and pressure-monitoring devices. It provides oxygen delivery to the blood, carbon dioxide removal from the blood and maintenance of perfusion and body temperature.

\section{Venous cannulation and drainage}

Basic approaches for central venous cannulation include bicaval, single atrial, or cavo-atrial (two stage). Venous blood usually enters the circuit by gravity or siphonage into a venous reservoir placed 40 to $70 \mathrm{~cm}$ below the level of the heart. Most aortic valve and coronary artery surgeries can be done with single venous cannulation. Cavo-atrial cannula ("two-stage") provides better drainage than a single cannula (narrowed distal end is threaded into the IVC while the wider proximalportion has side holes designed to rest within the right atrium. ${ }^{7}$ Femoral/ iliac vein cannulation is mostly indicated for redo operations, aortic and thoracic surgery to prevent bleeding complications during sternotomy. ${ }^{8}$

Venous cannulation and drainage is associated with complications like arrhythmias, bleeding, air embolism, injury or obstruction due to catheter malposition, and decannulation if not secured properly.

\section{Venous reservoir}

Venous reservoir (volume reservoir) is placed before the arterial pump. Reservoirs may be rigid (hard) plastic canisters ("open” types) or soft, collapsible plastic bags ("closed" types). ${ }^{9}$

Soft bag reservoirs reduces the risk of pumping massive air emboli by eliminating the blood-gas interface.

\section{Arterial cannulation}

Arterial catheters are rated by a performance index which is based on external diameter, flow, and pressure differential. Cannulation sites include the proximal aorta, innominate 
artery and distal arch, and femoral, external iliac, axillary and subclavian arteries.

Complications associated are difficulty in insertion, bleeding, aortic wall tear, malpositioning, atheromatous or air embolism, obstruction to flow (high line pressure), inadequate or excessive cerebral perfusion, inadvertent decannulation and aortic dissection.

\section{Oxygenators}

Membrane oxygenators replaces the lung function (Types: hollow fibers capillaries $(0.3-0.8 \mu \mathrm{m})$ of microporous polypropylene sheets or thin sheets of silicone rubber). Membrane oxygenators are safer than bubble oxygenators. Most units combine a venous reservoir, heat exchanger, and hollow- fiber membrane oxygenator into one compact unit. Flow regulators, flow meters, gas blender, oxygen analyzer, gas filter, and moisture trap are parts of the oxygenator gas supply system within membrane oxygenators. Often an anesthetic vaporizer is also added. Complications include oxygenator malfunction, leaks, loss of gas supply, rupture of connections, failure of the blender, and deteriorating gas exchange. ${ }^{10}$

\section{Heat exchangers}

During CPB, body temperature is controlled with the help of heat exchanger by heating or cooling blood passing through the perfusion circuit. Hypothermia isused more frequently during cardiac surgery to reduce oxygen demand or to facilitate anesthesia for open heart surgery operative exposure by temporary circulatory arrest. Complication include leakage of water into the blood path can cause hemolysis and malfunction of heater/cooler units.

\section{Pumps}

Most heart-lung machines use two types of pumps; centrifugal pumps and roller pumps.

Roller pumps consist of polyvinyl, silicone, or latex tubing, which is compressed by two rollers $180^{\circ}$ apart inside a curved raceway. ${ }^{11}$ Flow rate is determined from calibration curves for each pump for different tubing sizesand rates of rotation. Roller pumps are inexpensive, reliable, safe, insensitive to afterload, and have small priming volumes, but can produce high negative pressures and microparticles shed from compressed tubing (spallation). Roller pumps are also used for sucker systems and for delivering cardioplegic solutions.

Centrifugal pumps consist of a vaned impeller or nested, smooth plastic cones, which when rotated rapidly, propel blood by centrifugal force. ${ }^{12}$ An arterial flow meter is required to determine forward blood flow, which varies with the speed of rotation and the afterload of the arterial line. Centrifugal pumps are probably superior for temporary extracorporeal assist devices and left heart bypass, and for generating pump-augmented venous return. Complications include loss of electricity, loss ability to control pump speed also called pump creep when turned off, flow meter or RPM indicator malfunction, rupture of tubing and improper tubing in the raceway causing flow. Manual pumping option should be available in case of electrical failure.

\section{Cardioplegia delivery systems}

Cardioplegic solutions are delivered through a separate perfusion system that includes a reservoir, heat exchanger, roller pump, bubble trap and microfilter.

\section{Venting the heart}

If the heart is unable to contract, distention of either ventricle is detrimental to subsequent contractility. Methods for venting the heart are aortic root vent, catheter placed in the LV through right superior pulmonary vein/left atrial junction, direct venting of the $L V$ at the apex, venting the main pulmonary artery.

\section{Tubing and connectors}

The various components of the heart-lung machine are connected by polyvinyl tubing and fluted polycarbonate connectors. Heparin can be attached to blood surfaces of all components of the extracorporeal circuit by ionic or covalent bonds. Use is limited because of their questionable efficacy, cost and limited experience.

\section{Filters and bubble traps}

Two types of blood micro-filters are available for use within the perfusion circuit; Depth (packed fibers or porous foam) and Screen (woven polyester or nylon thread and have a defined pore size, and filter by interception). ${ }^{13}$ Filters are located in arterial line, reservoir, cardiotomy suction and cardioplegia delivery system.

\section{Assembly of the heart-lung machine}

Adult extracorporeal perfusion circuits require 1.5 to $2.0 \mathrm{~L}$ of balanced electrolyte solution (lactated Ringer solution, Normosol-A, or Plasma-Lyte). Before connections are made to the patient, the prime is recirculated through a micropore filter to remove particulate matter and air.

\section{References}

1. Nashef SA, Roques F, Michel P, Gauducheau E, Lemeshow S, Salamon R et al. European system for cardiac operative risk evaluation (EuroSCORE). Eur J Cardiothorac Surg 1999;16(1):9-13.

DOI: 10.1016/S1010-7940(99)00134-7

2. Dupuis JY, Wang F, Nathan H, Lam M, Grimes S, Bourke M. The Cardiac Anesthesia Risk Evaluation score: a clinically useful predictor of mortality and morbidity after cardiac surgery. Anesthesiology 2001;94:194-204.

3. Kloner RA. Consequences of brief ischaemia: stunning, preconditioning, and their clinical implications. Circulation 2001;104:3158-3167.

DOI: 10.1161/hc5001.100039

4. Sandham JD, Hull RD, Brant RF, Knox L, Pineo GF, Doig CJ et al. A randomized, controlled trial of the use of pulmonary-artery catheters in high risk surgical patients. N Engl J Med 2003;348:5-14.

DOI: 10.1056/NEJMoa021108

5. Thys D. Practice guidelines for perioperative transesophageal echocardiography, A report by the American Society of Anesthesiologists and the Society of Cardiovascular Anesthesiologist Task Force on Transesophageal Echocardiography. Anesthesiology 1996;84:986-1006. DOI: 10.1097/ ALN.0b013e3181c51e90

6. Lehmann A, Karzau J, Boldt J, Thaler E, Lang J, Isgro F. Bispectral Index-guided anaesthesia in patients undergoing aortocoronary bypass grafting. Anesth Analg 2003;96:336-43.

DOI: 10.1213/00000539-200302000-00008 
7. Arom KV, Ellestad C, Grover FL, Trinkle JK. Objective evaluation of the efficacy of various venous cannulas. J Thorac Cardiovasc Surg 1981;81:464.

8. Merin O, Silberman S, Brauner R, Munk Y, Shapira N, Falkowski $\mathrm{G}$ et al. Femoro-femoral bypass for repeatopen-heart surgery. Perfusion.

9. Fisher AR. The incidence and cause of emergency oxygenator changeovers. Perfusion 1999;14:207-12. DOI: 10.1177/026765919901400309

10. Stammers AH. Extracorporeal devices and related technologies, in Kaplan JA (ed): Cardiac Anesthesia, 4th ed. Philadelphia, WB Saunders 1999, P1018.

11. Leschinsky BM, Itkin GP, Zimin NK. Centrifugal blood pumps - a brief analysis: development of new designs. Perfusion 1991;6:115-21.

12. Joffe D, Silvay G. The use of microfiltration in cardiopulmonary bypass. J Cardiothorac Vasc Anesth 1994;8(6):685-92. 